\title{
Bounds on Thermal Efficiency from Inference
}

\author{
Ramandeep S. Joha ${ }^{*}$ \\ Department of Physical Sciences, Indian Institute of Science Education and Research Mohali, \\ Sector 81, S.A.S. Nagar, Manauli P.O., Mohali 140306, India \\ Renuka Rai \\ Department of Applied Sciences, University Institute of Engineering \\ and Technology, Panjab University, Chandigarh-160014, India. \\ Günter Mahler \\ Universität Stuttgart, 1. Institut für Theoretische Physik, \\ Pfaffenwaldring 57 // IV, 70550 Stuttgart, Germany
}

\begin{abstract}
The problem of inference is applied to the process of work extraction from two constant heat capacity reservoirs, when the thermodynamic coordinates of the process are not fully specified. The information that is lacking, includes both the specific value of a temperature as well as the label of the reservoir to which it is assigned. The estimates for thermal efficiency reveal that uncertainty regarding the exact labels, reduces the maximal efficiency below the Carnot value, its minimum value being the well known Curzon-Ahlborn value. We also make an average estimate of the efficiency before the value of the temperature is revealed. It is found that if the labels are known with certainty, then in the near-equilibrium limit the efficiency scales as $1 / 2$ of Carnot value, while if there is maximal uncertainty in the labels, then the average estimate for efficiency drops to $1 / 3$ of Carnot value. We also suggest how infered properties of the incomplete model can be mapped to a model with complete information but with an additional source of thermodynamic irreversibility.
\end{abstract}

PACS numbers: 05.70.a, 05.70.Ln, 02.50.Cw

Keywords: Inference; Uncertainty; Thermal efficiency; Irreversibility

\section{INTRODUCTION}

In many situations, we have to reason from incomplete information. Scientific inference refers to application of a consistent set of principles in these situations, which satisfy our rationality. In its initial stages, it was termed as the "the art of conjecturing" by Bernoulli [1. Later refined into a technical tool, Laplace 2 made a successful use of what is now known as Bayes' formula [3. Later, Cox showed that the only set of consistent axioms justifying plausible reasoning were the already established axioms of the probability theory 4, 5. Many authors have clarified the scope and meaning of inference [6 9]. Following Jaynes, much of the development in thermodynamics and statistical mechanics may be regarded as an application of the principles of plausible reasoning.

Usually, the physical mechanisms which generate thermodynamic irreversibility are ascribed to a finite rate of heat transfer, internal friction, the finite size of the reservoirs and so on. However, from the perspective of information theory, this irreversibility is also related to a loss of information about the system into the environment. In this paper, we consider reversible thermodynamic models but with incomplete information. The issue we address here is that from inference performed on such models, the estimated behavior exhibits some features of irreversibility. Thus in our case, irreversibility does not appear at

\footnotetext{
* Corresponding Author: rsjohal@iisermohali.ac.in
}

the objective physical level, but only in the estimated behavior. The central feature of our approach is that the missing part of information is interpreted in a subjective manner, or in other words as the observer's lack of knowledge about the system. Due to lack of complete information, the observer has to perform inference to estimate the characteristic quantities of the system. We show that a consistent use of prior information in reversible models leads to an estimate for maximal efficiency which is lower than the Carnot value. We also suggest that the resulting inferred behavior is analogous to that obtained by incorporating explicitly some thermodynamic irreversibility in the actual (reversible) process.

The motivation for our approach comes from the connection between thermodynamics and information. This has prompted a fruitful discussion on the role of information theory in thermodynamic frameworks, for example the role of Maxwell's demon in information processing 10] which is continuing to this day. Recently, it has also been explored in 11 14, that the identification and inclusion of prior information in heat cycles with incomplete specification, leads to interesting analogies with irreversible models. In particular, many different efficiencies show up in the inference based approach which are found in the context of time-dependent cycles or with other sources of irreversibility [15].

Now the prior information, which is to be exploited in making inference, can be present in different forms, some even quite raw or qualitative. In this paper, we want to understand further how different kinds of prior 
information, impact our expectations about the performance of these heat cycles. In particular, we assume an uncertainty not only in the value of a parameter, but also an uncertainty in the exact subsystem to which it is assigned. The later kind of uncertainty will be addressed as label uncertainty. For example, a classical system may be specified by a set of quantities $\{X, Y, .$.$\} .$ In the case of a multi-partite system, we also label the subsystems, say with index $i$. Then the properties of all constituent subsystems are distinguished if our labels are refined as $\left\{X_{i}, Y_{i}, \ldots\right\}$. In the following, we consider a situation where the values of the individual parameters $(X, Y, .$.$) are known, but we may be uncertain about$ the exact subsystem labels. The question is how do we estimate the performance of the system based on this incomplete information. For simplicity, we will consider only a bipartite set up.

The paper is organised as follows. Section II discusses the model and has three subsections. In subsection A, we introduce a reversible thermodynamic model of work extraction, with complete specification of all parameters. In subsection B, we assume uncertainty in the final temperatures as well as the label uncertainty. The work performed and the thermal efficiency are estimated for a given measure of uncertainty. In subsection $\mathrm{C}$, we draw analogy of the infered behavior with an explicitly irreversible model of heat engine. Finally, in Section III we define an average estimate of efficiency, which is calculated using a uniform prior distribution over the uncertain temperature. The behavior of this average value for near-equilibrium is evaluated, which leads to establishing two distinct classes for the expected efficiency, based on zero or complete label uncertainty. The last section IV presents the conclusions.

\section{THE MODEL}

\section{A. The case of complete information}

It is sufficient to consider a textbook example of two finite ideal gas systems with a constant heat capacity $C$, at initial temperatures $T_{+}$and $T_{-}\left(T_{+}>T_{-}\right)$, serving as the heat source and the sink, respectively. They are coupled via a reversible work source, which by design extracts maximal work due to the available temperature gradient. At some stage in this process, the initially hot reservoir obtains a temperature $T_{1}$ and the initially cold reservoir is at temperature $T_{2}$. The amount of heat taken in by the engine and the heat rejected to the sink, are respectively given by $Q_{\text {in }}=C\left(T_{+}-T_{1}\right)$, and $Q_{\text {out }}=$ $C\left(T_{2}-T_{-}\right)$, respectively. The total entropy change in the two reservoirs being zero, we have $\triangle S=C \ln \left(T_{1} / T_{+}\right)+$ $C \ln \left(T_{2} / T_{-}\right)=0$. This yields

$$
T_{1}=\frac{T_{+} T_{-}}{T_{2}}
$$

as the relation between the final reservoir temperatures. The work performed, $W=Q_{\text {in }}-Q_{\text {out }}$ is: $W=C\left(T_{+}+\right.$ $\left.T_{-}-T_{1}-T_{2}\right)$. In the following, we set $C=1$. Finally, using Eq. (1), the efficiency $\eta=W / Q_{\text {in }}$, can be written as:

$$
\begin{aligned}
\eta & =1-\frac{T_{2}}{T_{+}}, \\
& =1-\frac{T_{-}}{T_{1}} .
\end{aligned}
$$

We note that the maximum work is obtained if the final temperatures obtained are: $T_{1}=T_{2}=\sqrt{T_{+} T_{-}}$, and the efficiency at this optimal process is $\eta=\eta^{*}=1-$ $\sqrt{T_{-} / T_{+}}$.

Now in the standard analysis, $T_{+}$and $T_{-}$are the fixed initial values of the temperatures, and due to relation (1), we may regard all the expressions as functions of only one of the two temperatures, $T_{1}$ or $T_{2}$. Thus the work performed, can be rewritten as

$$
W\left(T_{2}\right)=\left(T_{+}+T_{-}-T_{2}-\frac{T_{+} T_{-}}{T_{2}}\right),
$$

with a similar expression in terms of $T_{1}$.

Now note that just from the work expression, Eq. (4), it is not obvious as to which temperature is chosen as the variable. We have to look at the expression for the heat exchanged to assess the label corresponding to a specific reservoir. So when we have an exact knowledge about the temperatures, this information has two parts: i) the individual values of the temperatures and ii) the labels for the reservoirs to which a value is assigned. Thus the symbol $T_{2}$ denotes the temperature value of the particular reservoir (label 2).

\section{B. Incomplete information}

Now let us imagine a controller of the process who knows the final thermodynamic coordinates, or the temperatures of the reservoirs. The controller invites us to play a game of guessing and promises to reveal one of the values of the temperatures, but not the reservoir to which this value belongs. The task ahead of us is to infer the performance of the engine by making estimates about work performed, efficiency and so on.

As mentioned above, the work expression does not reveal unambiguously the individual labels of the reservoirs. Thus given some temperature value $T$, the work expression (written devoid of reservoir labels) will be $W(T)=T_{+}+T_{-}-T-T_{+} T_{-} / T$. Next comes the issue of the range of possible values for the final temperature $T$. This should be fixed from the information that is actually available. In particular, we invoke the fact that the extracted work satisfies: $W \geq 0$, so that $T$ is allowed to take values in the range $\left[T_{-}, T_{+}\right]$. Thus to some extent, we have removed the problem from its physical context which involves such notions as the flow of heat from a 
hot to cold temperature and so on. In the spirit of an inference based approach, we seek to quantify our beliefs focusing on the prior information.

To illustrate how our estimates are affected as our beliefs change, suppose further that we have a reason to believe that the disclosed value of temperature belongs to a specific reservoir. We quantify this belief by assigning a probability with numerical value $\gamma(0 \leq \gamma \leq 1)$ to the hypothesis that the disclosed value $T$ belongs to the initially hot reservoir (henceforth labeled A). The parameter $\gamma$ is assumed to be independent of the $T$ value.

Now we know that if one of the final temperatures is $T$, the corresponding value for the other reservoir definitely is $T_{+} T_{-} / T$. But as per our beliefs, the final temperature of reservoir A is: (i) $T$, with probability $\gamma$, and (ii) $T_{+} T_{-} / T$, with probability $1-\gamma$. Then upon knowing the value $T$, the expected final temperature of reservoir A may be defined as a weighted average over the two values:

$$
\bar{T}_{A}=\gamma T+(1-\gamma) \frac{T_{+} T_{-}}{T} .
$$

This is our estimate for the final temperature of reservoir $\mathrm{A}$, given the information that it is a maximum work process and, one of the final temperatures is $T$. Correspondingly, for reservoir B, we should have:

$$
\bar{T}_{B}=(1-\gamma) T+\gamma \frac{T_{+} T_{-}}{T} .
$$

Now we use these values to estimate further other quantities, which are relevant to the performance of the engine. Our estimate of the heat absorbed by the engine from reservoir $\mathrm{A}$, is given by

$$
Q_{\text {in }}=T_{+}-\bar{T}_{A} .
$$

Similarly, our estimate for the heat rejected to reservoir $\mathrm{B}$, will be:

$$
Q_{\text {out }}=\bar{T}_{B}-T_{-} .
$$

The estimate for work defined as: $W=Q_{\text {in }}-Q_{\text {out }}$, turns out to be $W=T_{+}+T_{-}-T-T_{+} T_{-} / T$, i.e. equal to the actual work performed. In particular, the estimate for work is independent of the parameter $\gamma$, showing that the work is not affected by label uncertainty.

For brevity, we now calibrate all the temperatures, relative to the initial temperature of reservoir $\mathrm{A}$, and define $\theta=T_{-} / T_{+}$. Then the expected work for a given value $T$, is

$$
W=1+\theta-T-\frac{\theta}{T} .
$$

Finally, we note that the estimate for the efficiency $\eta=$ $W / Q_{\text {in }}$, given by

$$
\eta_{\gamma}(T)=\frac{T+\theta T-T^{2}-\theta}{T-\gamma T^{2}-(1-\gamma) \theta},
$$

is also affected by the label uncertainty.

Let us now look at the behavior of the above efficiency for some special values of $\gamma$. When $\gamma=1(0)$, it corresponds to the case when we are certain that the disclosed value $T$ belongs to reservoir A (B). In these cases, Eq. (10) reduces to $\eta_{0}=1-T$ and $\eta_{1}=1-\theta / T$, respectively. On the other extreme, when we are maximally uncertain about the label for the temperature $T$, or which means equal probabilities for $T$ to belong to any of the two reservoirs, then we must assign $\gamma=1 / 2$. Then from Eq. 110, the efficiency is expected to be

$$
\eta_{\frac{1}{2}}(T)=\frac{2\left(T+\theta T-T^{2}-\theta\right)}{\left(2 T-T^{2}-\theta\right)} .
$$

Now there are two quantities of interest:

a) Maximum work: this is obtained by setting $\partial W / \partial T=0[16$, which holds at $T=\sqrt{\theta}$. The efficiency at maximum work is the well-known Curzon-Ahlborn formula: $\eta_{\mathrm{CA}}=1-\sqrt{\theta}$.

b) Estimated maximum efficiency: this is given by the condition: $\partial \eta_{\gamma} / \partial T=0$. From Eq. (10), the maximum value is:

$$
\eta_{\gamma}^{*}=\frac{1-\theta}{1+\sqrt{4 \gamma(1-\gamma) \theta}} .
$$

From Eq. 12, we see that in both cases of certainty

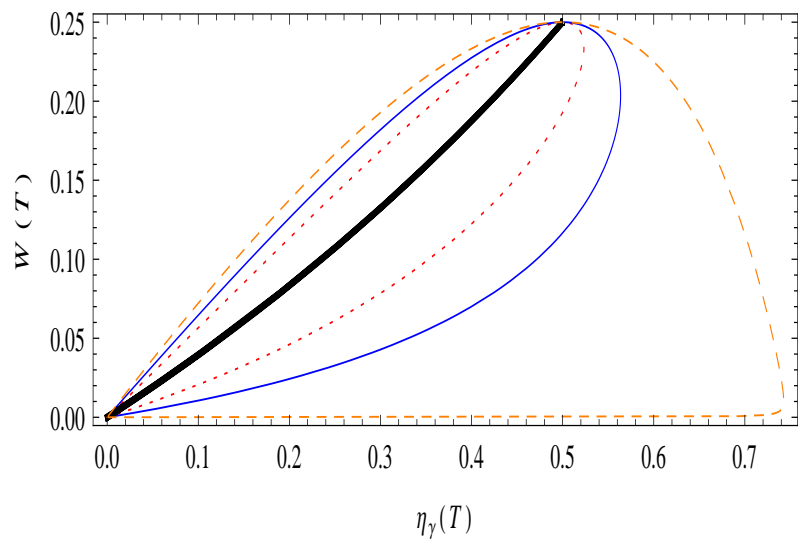

FIG. 1. A parametric plot between $W(T)$ as in Eq. 9 and efficiency in Eq. 10 , for $\theta=0.25$. $T$ takes values in the range $[\theta, 1]$ and $\gamma$ is kept fixed: $1 / 2$ (thick black, monotonic curve), $1 / 4$ (dotted), 1/8 (thin), and 1/10000 (dashed). The point for maximum work remains the same, while the maximum in the efficiency shifts with $\gamma$. There is a finite work obtained at the maximum of efficiency, which reduces and goes to zero as $\gamma \rightarrow 0$ (or $\gamma \rightarrow 1$ ), whereby the maximum efficiency reaches Carnot value, $1-\theta=0.75$.

about the labels, implying maximal information, the maximum efficiency is the Carnot value. But an uncertainty in the exact labels reduces the maximal efficiency $\left(\eta_{\gamma}^{*}\right)$ below the Carnot value, and it reduces to $\mathrm{CA}$ value, in the case of maximal uncertainty $(\gamma=1 / 2)$. Thus the upper bound for efficiency is related here directly with 


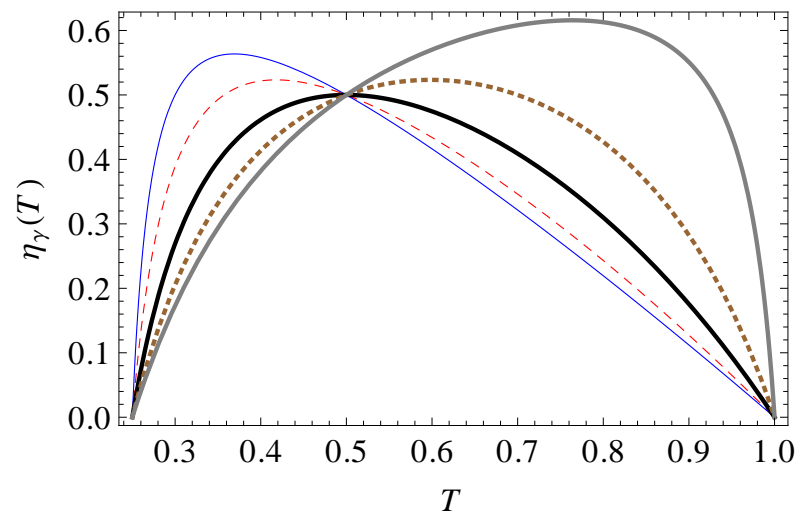

FIG. 2. Plot of Eq. 10 for $\theta=0.25$. $T$ takes values in the range $[\theta, 1] . \gamma$ varies as $1 / 2$ (thick black), $1 / 4$ (dashed), $1 / 8$ (thin), $3 / 4$ (dotted) and 19/20 (thick gray). The maximum value of efficiency for a given $\gamma$ is given by Eq. (12). As $\gamma \rightarrow 0$ or $\gamma \rightarrow 1$, the maximal value approaches the carnot value, $1-\theta=0.75$.

our state of knowledge and the CA efficiency emerges from an entirely different perspective. Here we have a mechanism which shows how inference under incomplete information leads us to expect a lower value for the maximum efficiency obtainable from a thermal engine. This feature is further exhibited in Fig. 11, which shows the work versus efficiency curves, each with a fixed value of $\gamma$. In general, the maximum work and the maximum efficiency points are different. At $\gamma=0$ and $\gamma=1$, they are maximally separate, but tend to merge together as $\gamma \rightarrow 1 / 2$.

\section{Analogy with irreversible model}

The fact that the maximal efficiency drops below the Carnot efficiency, indicates that we are infering an irreversible behavior for an otherwise reversible model with incomplete information. In this section, we suggest an irreversible physical process which corresponds to the above picture obtained through inference.

The reservoirs $\mathrm{A}$ and $\mathrm{B}$ are initially at (scaled) temperatures 1 and $\theta$, respectively. Now imagine a two-step process. In the first step, work is extracted by coupling reservoirs with a reversible work source, so that at the end of this step, reservoir A reaches temperature $T$ and consequently, reservoir $\mathrm{B}$ is at temperature $\theta / T$. The work performed is given by Eq. (9). In the second step, the two reservoirs are detached from the work source and put in mutual thermal contact. Heat is exchanged between them, conserving their total internal energy. Let at the end of the second-step, the temperatures of the reservoirs be $\bar{T}_{A}$ and $\bar{T}_{B}$, as given by Eqs. $(5)$ and $(6)$. The second step is intrinsically irreversible. The net heat released by reservoir A is: $Q_{\text {in }}=1-\bar{T}_{A}$ and the net heat rejected to reservoir B is: $Q_{\text {out }}=\bar{T}_{B}-\theta$. These quantities are the same as obtained through inference in Section
II.B.

In the above two-step process, we are assigning definite temperatures to the reservoirs $\mathrm{A}$ and $\mathrm{B}$. Due to the second law, we expect that overall the heat flows from hot to cold reservoir. So at the end of work extracting process, we require that

$$
T \geq \theta / T
$$

If $T=\theta / T$, then the second step is redundant. So if $T>\theta / T$ holds then by the end of second step, we also expect that

$$
\bar{T}_{A} \geq \bar{T}_{B}
$$

It can be easily seen that the above condition requires $\gamma \geq 1 / 2$, with $\bar{T}_{A}=\bar{T}_{B}$ implying $\gamma=1 / 2$.

\section{AVERAGE ESTIMATE OF EFFICIENCY}

So far, we have assumed that the values of final temperatures are pre-specified. For instance, Eq. (11) provides an estimate of efficiency for a given value $T$ but with complete ignorance about the reservoir labels. If we are only provided the value $T$, then we must take $\gamma=1 / 2$ and our estimate for efficiency will be Eq. (11). In this section, we extend the game of guessing further and estimate the efficiency before we are provided the value $T$. So now we assume to be ignorant about the value $T$ also. To quantify our guess in the absence of the value $T$, we have to specify a prior distribution for $T$ [13, 14, which takes into account our belief as to which value $T$ from the allowed range, the controller may be holding. If we do not have a reason to expect one value over another, then all allowed values are equally likely in the interval $\left[T_{-}, T_{+}\right] \equiv[\theta, 1]$. So we must adopt a uniform prior distribution for $T$. Our average estimate for efficiency, defined as the mean value over this uniform prior is then

$$
\bar{\eta}_{\frac{1}{2}}(\theta)=\int_{\theta}^{1} \eta_{\frac{1}{2}}(T) \frac{d T}{1-\theta} .
$$

On using Eq. (11) and solving Eq. (15), we obtain

$$
\begin{aligned}
\bar{\eta}_{\frac{1}{2}}= & 2-\left(1+\frac{1}{\sqrt{1-\theta}}\right) \ln (1+\sqrt{1-\theta}) \\
& -\left(1-\frac{1}{\sqrt{1-\theta}}\right) \ln (1-\sqrt{1-\theta}) .
\end{aligned}
$$

For close to equilibrium situations, i.e. $1-\theta \approx 0$, the average value of efficiency behaves as follows

$$
\bar{\eta}_{\frac{1}{2}}=\frac{1-\theta}{3}-\frac{(1-\theta)^{2}}{10}+O[1-\theta]^{3} .
$$

On the other extreme, consider the two cases of certainty about the reservoir labels. For the special case of $\gamma=0$, 
we have $\eta_{0}(T)=1-T$, whose average over the uniform prior is

$$
\bar{\eta}_{0}=\frac{1-\theta}{2} .
$$

Also for $\gamma=1$, we have $\eta_{1}(T)=1-\theta / T$ and the average value over uniform prior, is given by

$$
\bar{\eta}_{1}=1+\frac{\theta \ln \theta}{1-\theta},
$$

whose expansion behaves as: $\bar{\eta}_{1} \approx(1-\theta) / 2+(1-\theta)^{2} / 6+$ $O[1-\theta]^{3}$. Thus, both of the above averages yield that for near-equilibrium conditions, the average efficiency is given by $(1-\theta) / 2$. This result holds if the reservoir labels are known i.e. we know which reservoir temperature is chosen as the uncertain variable. In contrast, we obtain one-third of Carnot value, if we are maximally uncertain about the specific reservoir labels. This is the main result of the paper, that the expected efficiency near equilibrium falls under two different classes, determined by the state of our knowledge about the system. Fig. 3 shows this dependence on $\gamma$ in a more clear fashion.

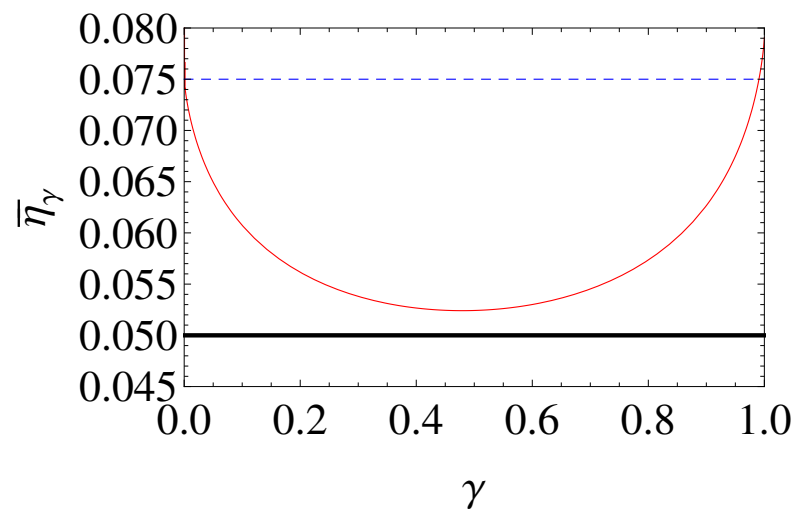

FIG. 3. Plot of the average efficiency over uniform prior versus $\gamma$, for $\theta=0.85$. The horizontal lines denote $(1-\theta) / 2$ and $(1-\theta) / 3$ which act as lower bounds for the case $\gamma=1$, and $\gamma=1 / 2$, respectively. For $\gamma=0$, the efficiency estimate is exactly $(1-\theta) / 2$.

\section{SUMMARY}

Inference is a kind of common sense reasoning in the face of incomplete information. It seeks to provide the most unbiased guess consistent with the constraints and laws obeyed by the system. We have considered a plausible use of this reasoning for classical thermodynamic machines operating under reversible conditions. An important new kind of uncertainty studied in this paper relates to incomplete knowledge about the labels of the uncertain parameters. We define a guessing game which combines features of subjective ignorance with the objective matters of fact about the physical system. Some interesting observations made are that the infered behavior has features of irreversibility, for example, the maximum efficiency of the engine is below Carnot limit. We also suggested an analog physical model which mimics the estimates of thermodynamic quantities in the infered model. It is not known if the mapping to an actual physical model is always guaranteed from a given inference based model. Further, we cannot be sure about the uniqueness of this mapping, and there may exist more than one physical processes which simulate the consequences of our game, or conversely, more than one games which simulate a physical process. However, in our opinion, the possibility of a mapping to an objective physical model indicates that inference anticipates a behavior which is allowed by the physical laws. Thus the mapping also reassures that the estimates are consistent with the laws of thermodynamics.

We have estimated an average efficiency based on the prior probability distribution and found that the so called label uncertainty makes the efficiency drop to $1 / 3$ of Carnot value in near-equilibrium regime. On the other hand, if there is no label uncertainty, the infered efficiency is equal to $1 / 2$ of Carnot value. This is consistent with our expectation that more information we lack, less is the efficiency we expect. Still the goal of inference is not to predict the "true" behavior of physical models proper, but to make a rational guess based on incomplete information.

In this paper we have defined a set of specific "games" representing additional subjective lack of information. This setting supplements the "objective ignorance" present already due to the laws of thermodynamics. Nevertheless, it turns out that the combined model may be equivalent to a "purely physical" model with some specific irreversible features - a remarkable non-uniqueness in terms of interpretation. It is hoped that the approach and conclusions of this paper may help to further develop procedures which incorporate different kinds of prior information, which then could shed light on the subtle interplay between subjective and objective ignorance presumed in the modeling of natural phenomena like, e.g., in the context of (rather complex) climate models.
[1] J. Bernoulli, The Art of Conjecturing, together with Letter to a Friend on Sets in Court Tennis, translated by Edith Dudley Sylla, Johns Hopkins University Press
(2006) Baltimore.

[2] P.S. Laplace, Memoir on the Probabilities of the Causes of Events, translated by S. M. Stigler, Stat. Sc. 1, 364 
(1986)

[3] T. Bayes, Phil. Trans. Roy. Soc. 330 (1763).

[4] R. T. Cox, Am. J. Phys. 17, 1 (1946).

[5] R.T. Cox, Algebra of Probable Inference, The Johns Hopkins University Press (2001).

[6] H. Jeffreys, Scientific Inference, Cambridge University Press (1931).

[7] H. Jeffreys, Theory of Probability, Second edition, Clarendon Press, Oxford (1948).

[8] G. Polya, Mathematics and Plausible Reasoning, Vol. I and II, Princeton University Press (1954).

[9] E. T. Jaynes, Probability Theory: The Logic of Science (Cambridge University Press, Cambridge, 2003).

[10] H. S. Leff and A. F. Rex, Maxwell's Demon 2: Entropy, Classical and Quantum Information, Computing (Institute of Physics, Bristol, 2003).
[11] R.S. Johal, Phys. Rev. E 82041119 (2010).

[12] G. Thomas and R.S. Johal, Phys. Rev. E 85041146 (2012).

[13] P. Aneja and R.S. Johal, Proceedings of Sigma-Phi International Conference on Statistical Physics-2011, Cent. Eur. J. Phys. 10 (3) 708 (2012).

[14] G. Thomas, P. Aneja and R.S. Johal, Proceedings of International Conference on Frontiers of Quantum and Mesoscopic Thermodynamics-2011, Phys. Scr. T151 014031 (2012).

[15] See 11, 13] and references therein.

[16] H.B. Callen, Thermodynamics and an Introduction to Thermostatistics, Second edition, John Wiley \& Sons (1985). 